\title{
Rational Design of Inverted Nanopencil Arrays for Cost-Effective, Broadband and Omnidirectional Light Harvesting
}

Hao Lin ${ }^{1, \dagger}$, Fei Xiu ${ }^{1,4, \dagger}$, Ming Fan ${ }^{l}$, SenPo Yip ${ }^{1,4}$, HoYuen Cheung ${ }^{2}$, Fengyun Wang ${ }^{3}$, Ning Han $^{1,4}$, Kwok Sum Chan ${ }^{1,4,5}$, Chun-Yuen Wong ${ }^{2}$, Johnny C. Ho ${ }^{1,4,5^{*}}$

${ }^{1}$ Department of Physics and Materials Science, City University of Hong Kong, 83 Tat Chee Avenue, Kowloon, Hong Kong

${ }^{2}$ Department of Biology and Chemistry, City University of Hong Kong, 83 Tat Chee Avenue, Kowloon, Hong Kong

${ }^{3}$ Cultivation Base for State Key Laboratory, Qingdao University, No. 308 Ningxia Road, Qingdao, People's Republic of China

${ }^{4}$ Shenzhen Research Institute, City University of Hong Kong, Shenzhen, People's Republic of China

${ }^{5}$ Centre of Functional Photonics (CFP), City University of Hong Kong, Tat Chee Avenue, Kowloon, Hong Kong

${ }^{\dagger}$ These authors contributed equally to this work.

*Corresponding author: johnnyho@cityu.edu.hk

SUPPORTING INFORMATION 
Figure S1

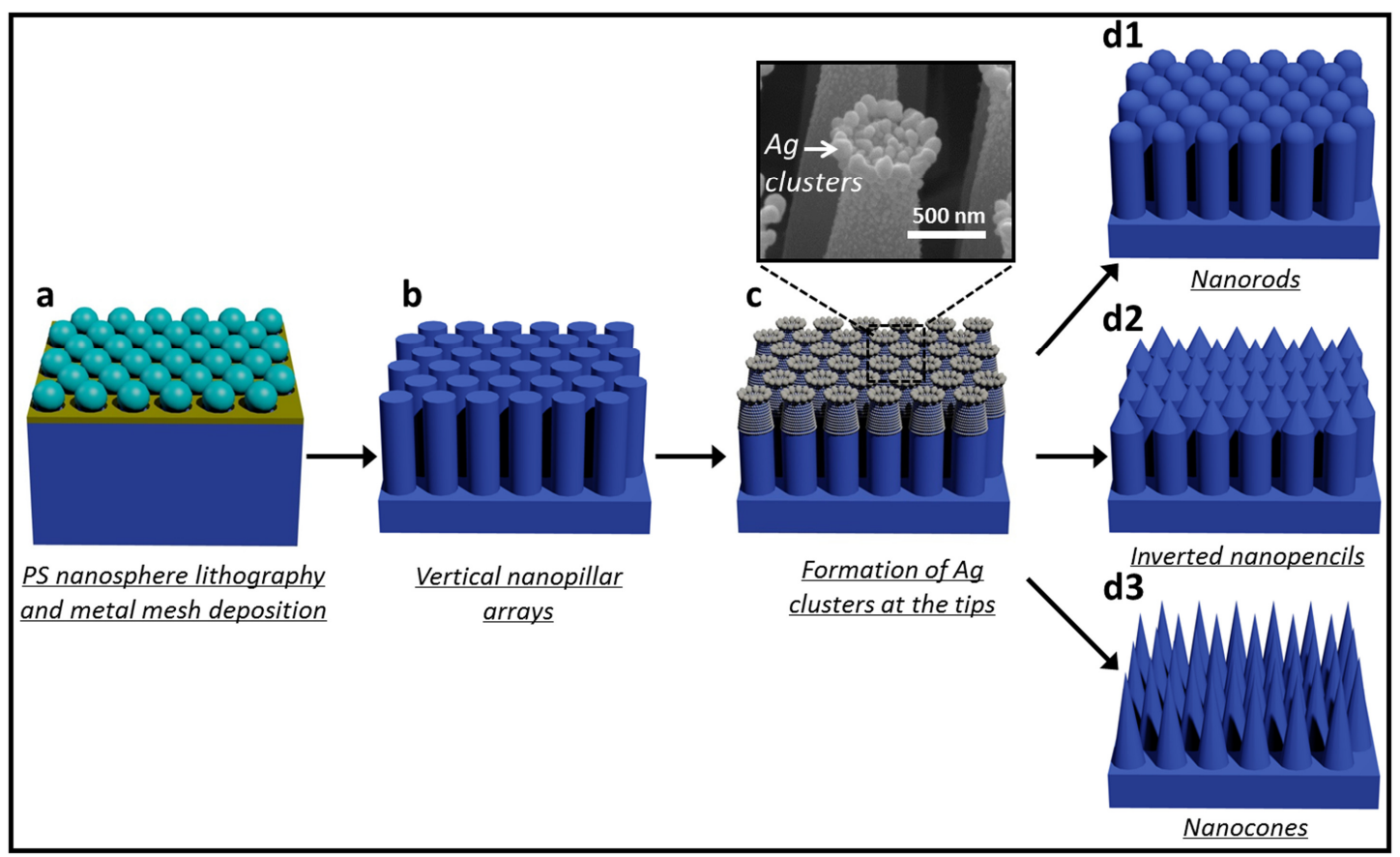

Figure S1. Process schematics of the formation of different morphological Si nano-arrays via the anisotropic wet etching technique, including nanopillars, nanorods, inverted nanopencils and nanocones. The inset shows the zoom-in SEM image of the nanopillar tips, demonstrating the selective deposition of Ag nanoclusters (on the tips only but not on the base). 
Figure S2

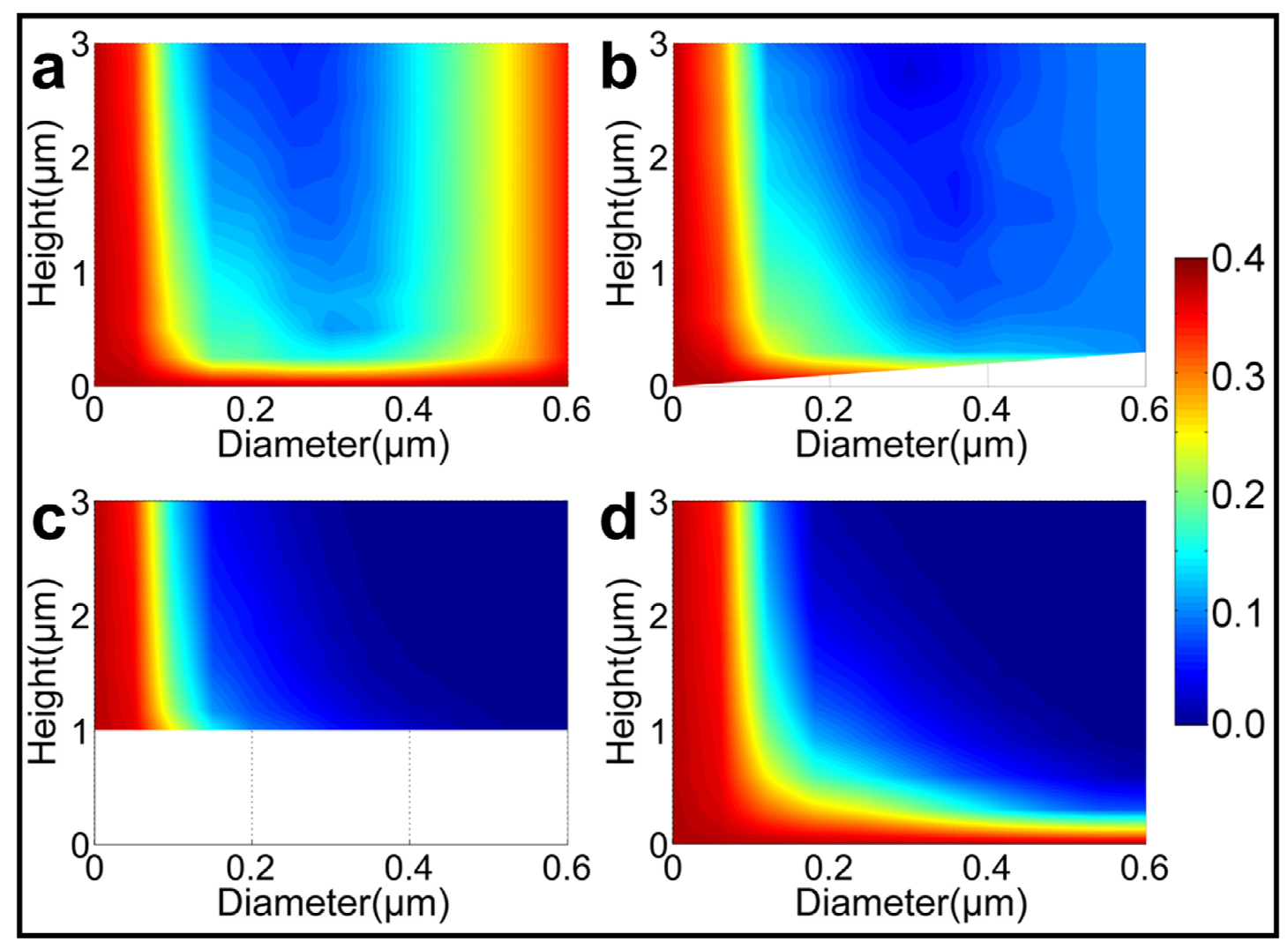

Figure S2. 2D simulated contours of broadband-integrated reflectance of various nanostructures with the pitch of $0.6 \mu \mathrm{m}$. (a) nanopillars, (b) nanorods, (c) inverted nanopencils and (d) nanocones. 
Figure S3

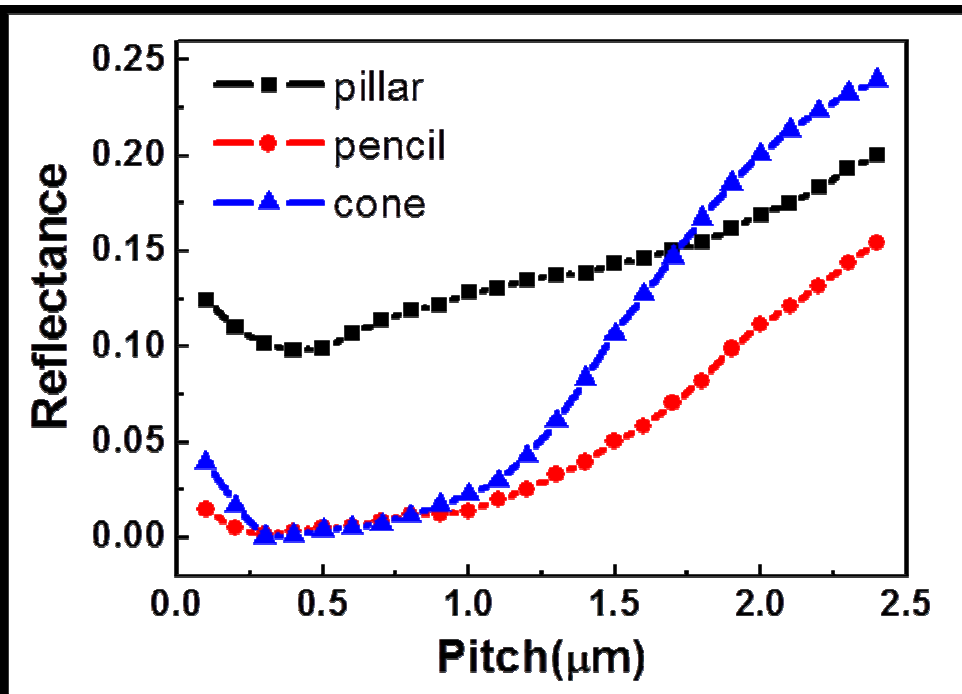

Figure S3. Simulated broadband-integrated reflectance of nanopillars, nanopencils and nanocones with different pitch. The material filling (base-diameter-to-pitch) ratio is fixed at 0.6 while the pillar height is fixed at $2 \mu \mathrm{m}$.

Basically, we find that for nanostructures with the pitch larger than $0.8 \mu \mathrm{m}$, the reflectance would increase accordingly due to the increased reflection from the bottom surface of these nanostructures. For the pitch between 0.3 to $0.8 \mu \mathrm{m}$, the reflectance stays relatively low, smaller than $1 \%$, owing to the efficient coupling of light into the nanostructures for the excellent absorption. When the pitch is below 0.3 $\mu \mathrm{m}$, since the gap between nano-arrays becomes so small, there is no more coupling and propagation effect of light into the basal region; therefore, most of the light would just get bounced back for the increased reflection. 
Figure S4

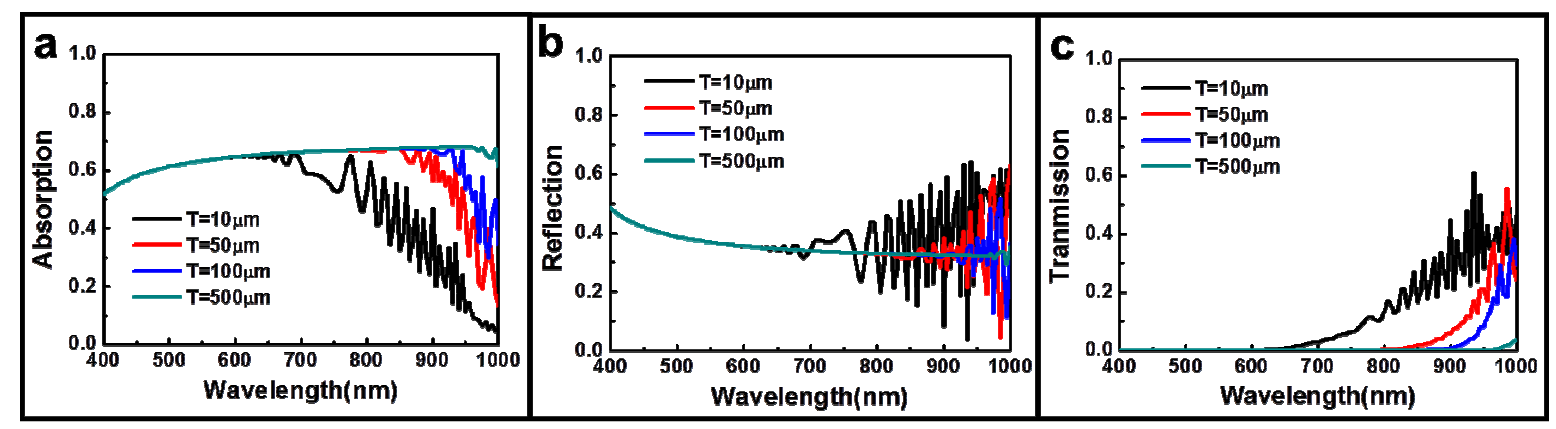

Figure S4. Simulation of optical properties for planar silicon with different substrate thickness. (a) Absorption, (b) reflection and (c) transmission. 
Figure S5

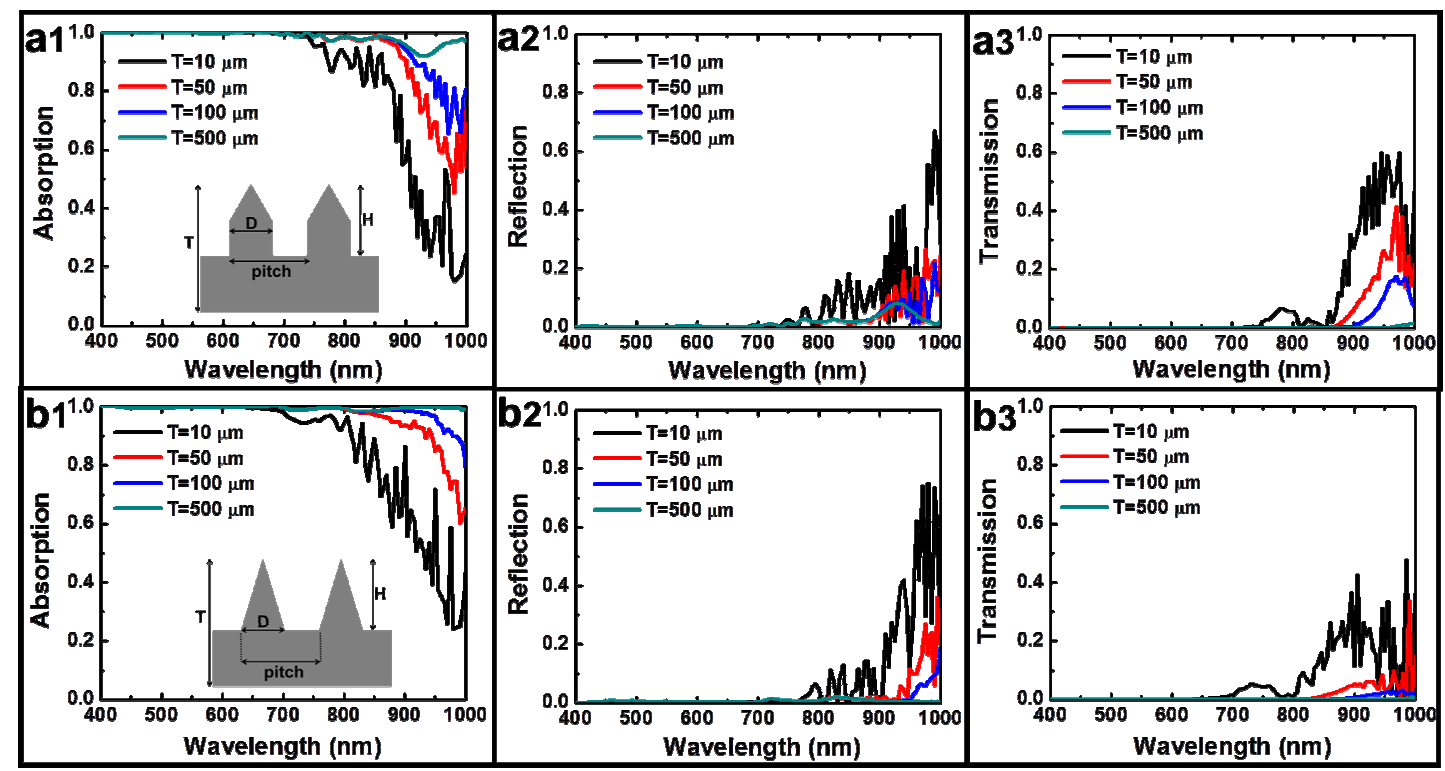

Figure S5. Simulation of optical properties for inverted nanopencils and nanocones with different substrate thickness. (a1) Absorption, (a2) reflection and (a3) transmission data of nanopencils with the pitch of $0.6 \mu \mathrm{m}$, base diameter of $350 \mathrm{~nm}$, pillar height (H) of $2 \mu \mathrm{m}$ and pencil tip height (H1) of $1 \mu \mathrm{m}$. (b1) Absorption, (a2) reflection and (a3) transmission data of nanocones with the pitch of $0.6 \mu \mathrm{m}$, base diameter of $350 \mathrm{~nm}$ and pillar height $(\mathrm{H})$ of $2 \mu \mathrm{m}$. 
Figure S6

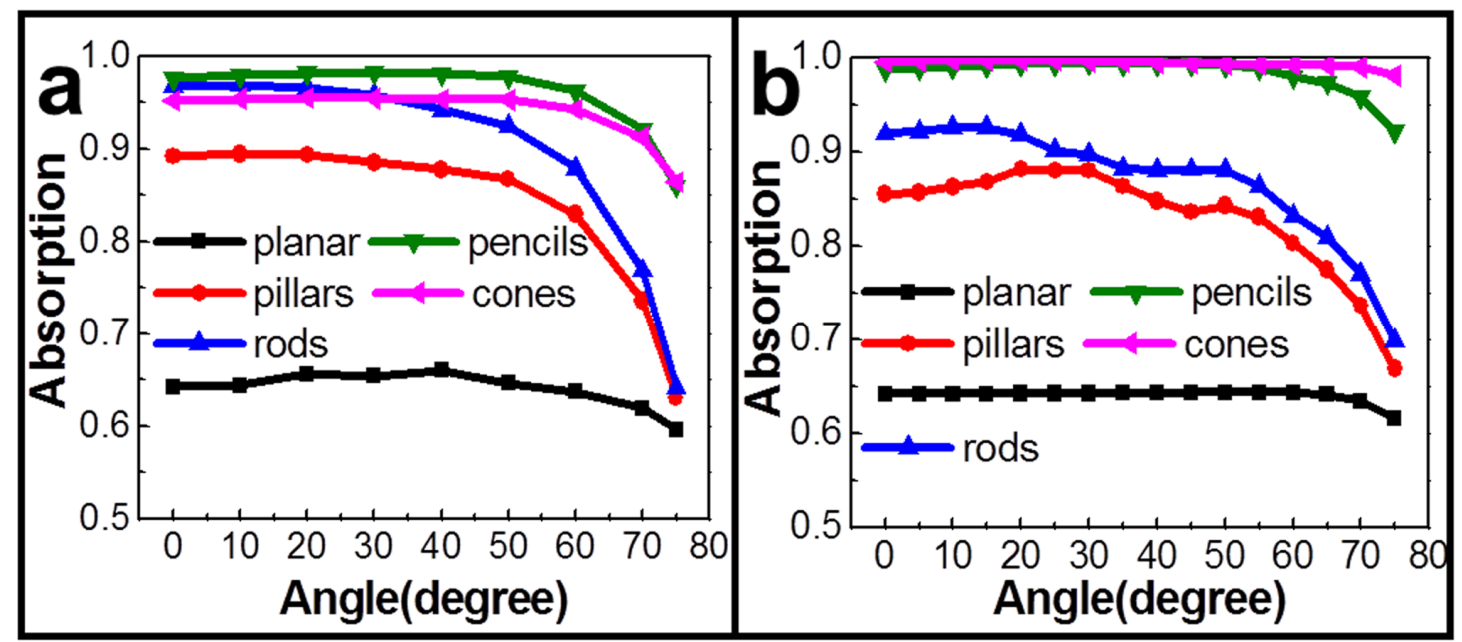

Figure S6. Comparison of the broadband-integrated absorption of different nanostructures with the pitch of $0.6 \mu \mathrm{m}$ and pillar height of $2 \mu \mathrm{m}$ between (a) experiment and (b) simulation. 
Figure S7

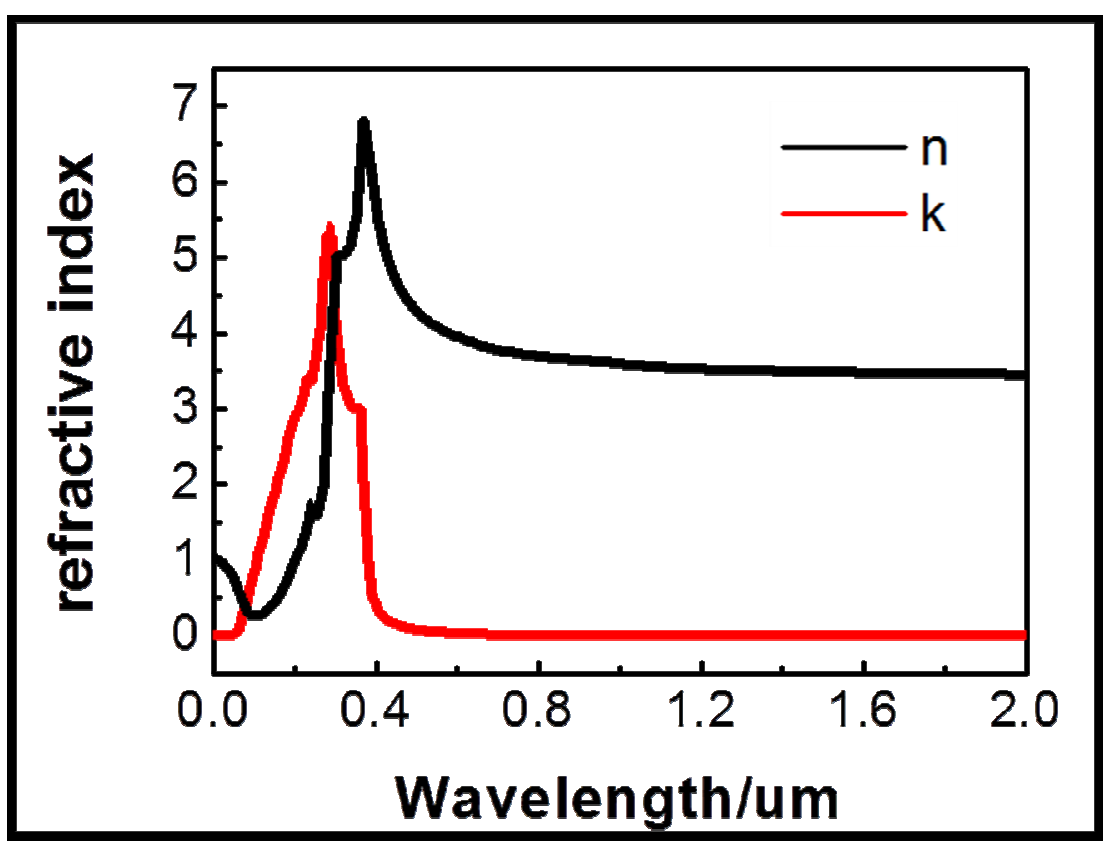

Figure S7. Plots of the refractive index values $\mathrm{n}$ and $\mathrm{k}$ of Si utilized in the simulations 
Figure S8

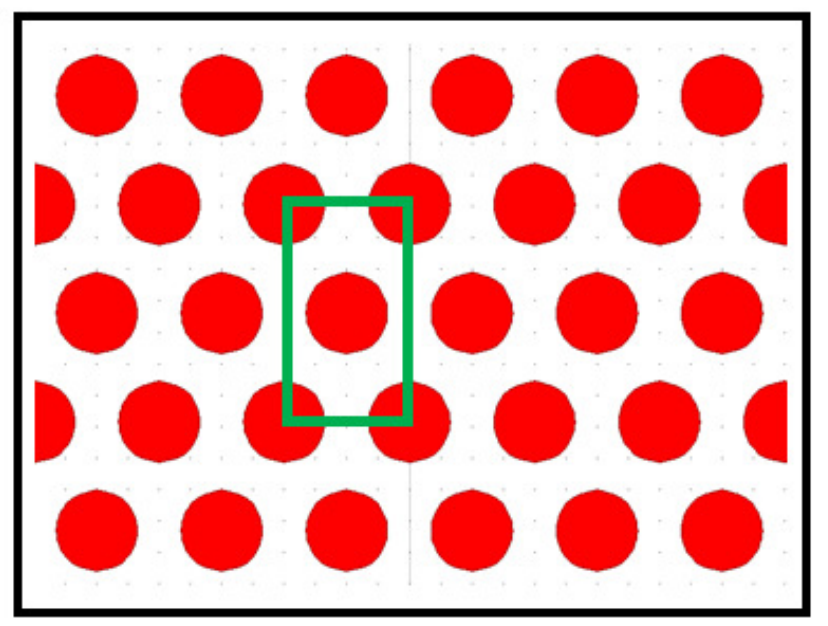

Figure S8. Schematic illustration of the unit cell (green line) performed in the simulations. 